Article

\title{
Linear Algorithms for Radioelectric Spectrum Forecast
}

\author{
Luis F. Pedraza 1,2,*, Cesar A. Hernandez ${ }^{1,2}$, Ingrid P. Paez ${ }^{2}$, Jorge E. Ortiz ${ }^{2}$ and \\ E. Rodriguez-Colina ${ }^{3}$
}

1 Faculty of Technology, Universidad Distrital Francisco José de Caldas, Bogota 110231, Colombia; cahernandezs@udistrital.edu.co

2 Industrial and Systems Engineering Department, Faculty of Engineering, Universidad Nacional de Colombia, Bogota 111321, Colombia; ippaezp@unal.edu.co (I.P.P.); jeortizt@unal.edu.co (J.E.O.)

3 Electrical Engineering Department, Universidad Autónoma Metropolitana Iztapalapa, Mexico City 09340, Mexico; erod@xanum.uam.mx

* Correspondence: lfpedrazam@udistrital.edu.co or lufpedrazama@unal.edu.co; Tel.: +57-1-323-93-00

Academic Editor: Javier Del Ser Lorente

Received: 2 August 2016; Accepted: 28 November 2016; Published: 2 December 2016

\begin{abstract}
This paper presents the development and evaluation of two linear algorithms for forecasting reception power for different channels at an assigned spectrum band of global systems for mobile communications (GSM), in order to analyze the spatial opportunity for reuse of frequencies by secondary users (SUs) in a cognitive radio (CR) network. The algorithms employed correspond to seasonal autoregressive integrated moving average (SARIMA) and generalized autoregressive conditional heteroskedasticity (GARCH), which allow for a forecast of channel occupancy status. Results are evaluated using the following criteria: availability and occupancy time for channels, different types of mean absolute error, and observation time. The contributions of this work include a more integral forecast as the algorithm not only forecasts reception power but also the occupancy and availability time of a channel to determine its precision percentage during the use by primary users (PUs) and SUs within a CR system. Algorithm analyses demonstrate a better performance for SARIMA over GARCH algorithm in most of the evaluated variables.
\end{abstract}

Keywords: cognitive radio; time series; linear algorithms

\section{Introduction}

Radioelectric spectrum occupancy is widely studied due to its importance for the construction of new spectrum assigning policies in emerging technologies, as well as in monitoring activities both in licensed and unlicensed bands. Real measurements for spectrum use within a determined band allow the corresponding authorities to guarantee that licenses meet local and regional spectrum regulations [1]. On the other hand, precise parameter estimates like time quantity and geographical region where the different spectrum band is actually used bring useful information to determine spectral opportunities for variant technologies within a domain. In this paper, such technologies correspond to a global systems for mobile communications (GSM) technology variant in the time domain [2].

The spectrum sensing in cognitive radio (CR) provides the necessary information about the status of the wireless channels, modeling and prediction of communications activity. This could contribute to spectral efficiency improvement efforts [3-5]. The prediction information of the channel status can be used by secondary users (SUs) to decide the sensing periods and channel occupancy duration for a single channel sensing scenario [6]. Besides, based on prediction information, SUs can select the channels with higher probability of vacancy in multi-channel wideband sensing scenarios [7], and also 
primary user (PU) occupancy models can be used as empty channel indicators replacing the spectrum sensing procedures $[2,8]$. A key issue for spectrum sensing operations is the evaluation of the so-called hidden node margin (HNM). The HNM has been evaluated in sharing scenarios between white space devices and digital terrestrial television systems [9].

Different initiatives for radioelectric spectrum channel modeling, mainly using deterministic and stochastic models, have been proposed [3,10-16]. Differences among those proposals and the present work lie in that in the first initiatives, the duty cycle (the average percentage of the channel utilization) time series is modeled for different types of channels, whereas the proposal presented here models the time series for received power in three GSM channels with different occupancy levels. For this purpose, firstly we used seasonal autoregressive integrated moving average (SARIMA) algorithm, which is adequate for analyzing time series with seasonality, in accordance with different studies [13-15]. Secondly, the generalized autoregressive conditional heteroskedasticity (GARCH) algorithm, which had been applied in traffic modeling and forecast for different communications networks, was also used [17-19].

The evaluation of the results obtained in algorithm forecasts is based on the following variables: channel availability time (the time interval where the channel is not used by the PUs), channel occupancy time (time interval where the channel is used by the PUs), observation time and error criteria analysis (symmetrical mean absolute percentage error, SMAPE, mean absolute percentage error, MAPE, and mean absolute error, MAE) [20-22].

\section{Theory and Background}

GARCH and SARIMA are deployed in a time series that is assumed as linear and with a known statistical distribution. As presented below, this is partially met in long-term analysis of time series measurements.

\subsection{Seasonal Autoregressive Integrated Moving Average Algorithm}

In general, if a time series exhibits potential seasonality indexed by $s$, then using a multiplied SARIMA $(p, d, q)(P, D, Q)$ s algorithm is advantageous, where $d$ is the level of non-seasonal differencing, $p$ is the autoregressive (AR) non-seasonal order, $q$ is the moving average (MA) non-seasonal order, $P$ is the number of seasonal autoregressive terms, $D$ is the number of seasonal differences, and $Q$ is the number of seasonal moving average terms. The seasonal autoregressive integrated moving average algorithm of Box and Jenkins [23] is given in the Equation (1),

$$
\varnothing_{p}(B) \Phi_{p}\left(B^{s}\right) \nabla^{d} \nabla_{s}^{D} x_{t}=\theta_{q}(B) \Theta_{Q}\left(B^{s}\right) e_{t}
$$

where $B$ is the backward shift operator, $x_{t}$ is the observed time series of load at time $t, e_{t}$ is the independent, identical, normally distributed error (random shock) at period $t ; \nabla_{s}^{D} x_{t}=\left(1-B^{s}\right)^{D} x_{t}$, $\Phi_{p}\left(B^{s}\right)$ and $\Theta_{Q}\left(B^{s}\right)$ are the seasonal $\operatorname{AR}(p)$ and $\operatorname{MA}(q)$ operators, respectively, which are defined in Equations (2) and (3),

$$
\begin{aligned}
\Phi_{p}\left(B^{s}\right) & =1-\Phi_{1} B^{s}-\Phi_{2} B^{2 s}-\ldots-\Phi_{p} B^{P s} \\
\Theta_{Q}\left(B^{s}\right) & =1-\Theta_{1} B^{s}-\Theta_{2} B^{2 s}-\ldots-\Theta_{Q} B^{Q s}
\end{aligned}
$$

where $\Phi_{1}, \Phi_{2}, \ldots, \Phi_{\mathrm{p}}$ are the parameters of the seasonal $\mathrm{AR}(\mathrm{p})$ model, $\Theta_{1}, \Theta_{2}, \ldots, \Theta_{Q}$ are the parameters of the seasonal MA(q) [24].

The Box-Jenkins methodology consists of four iterative steps [25]:

- $\quad$ Step 1: Identification. This step focuses on the selection of $d, D, p, P, q$ and $Q$. The number of the order can be identified by observing the sample autocorrelations (ACF) and sample partial autocorrelations (PACF).

- Step 2: Estimation. The historical data is used to estimate the parameters of the tentative model in Step 1. 
- Step 3: Diagnostic checking. Diagnostic test is used to check the adequacy of the tentative model.

- Step 4: Forecasting. The final model in Step 3 is used to forecast the values [26].

\subsection{Generalized Autoregressive Conditional Heteroskedastic Algorithm}

An important number of models, most of which have the property that conditional variance depends on past, have been proposed for capturing special data characteristics. Algorithms commonly used are those with autoregressive conditional heteroskedasticity $(\mathrm{ARCH})$ introduced in [27] and generalized ARCH (GARCH) given by [28]. Modeling ARCH-GARCH considers conditional error variance as a compression function of the past of the series.

ARCH modeling usually requires a great number of lags $(q)$, and therefore a great number of parameters. This might yield a model with a great number of parameters, which is in opposition to the parsimony principle. This fact drives many times to difficulties when using the model to describe data in an adequate way. On the contrary, a GARCH model uses an inferior quantity of parameters, which makes it preferable to an ARCH model [29-31]. In this paper, the GARCH algorithm with order $p \geq 0$ and $q \geq 0$ for the discrete-time stochastic process $r_{t}$ is expressed in Equations (4) and (5)

$$
\begin{gathered}
r_{t}=\mu+c, \varepsilon_{t} \sim N(0,1) \\
\sigma_{t}^{2}=\alpha_{0}+\sum_{i=1}^{q} \alpha_{i} r_{t-i}^{2}+\sum_{j=1}^{p} \beta_{j} \sigma_{t-j}^{2}
\end{gathered}
$$

where $\varepsilon_{t}$ is an independent and identically distributed process with a zero mean and one standard deviation, $\mu$ is the mean constant offset, $\sigma_{t}^{2}$ is variance, and $\alpha_{0}$ is the constant in the conditional variance. Unknown parameters for model are $\alpha_{0}, \alpha_{i}$ and $\beta_{j}$ for some positive integer $p, q$.

Just as in an ARIMA model, ACF and PACF are useful for $p$ and $q$ order identification in a $\operatorname{GARCH}(p, q)$ process [30].

\section{Case Study and Experiment Procedure}

The decision to carry out this study was made during the spectrum measurements campaign held in Bogota-Colombia where we obtained the measurements employed here from spectrum occupancy study previously carried out [1,32]. The band analyzed was the GSM $850 \mathrm{MHz}$, as it is a band constantly used and viable for analysis in time function with conventional equipment, like a spectrum analyzer. The measurements used in this study correspond to a week, from 23 December to 29 December 2012. In some studies [33], it has been indicated that a reasonable option to obtain representative data without any a priori information about a band is to consider measurement periods of at least $24 \mathrm{~h}$ in order to avoid under or overestimating frequency bands occupancy with some temporary patterns. While a 24-h measurement period could be thought of as adequate in order to properly characterize the activity of determined spectrum bands [34], in this research 7 days were analyzed, including patterns for workdays and weekends. Additionally, this time period is sufficient to measure occupancy in mobile networks with low use, as indicated in [2,34].

The channels to be modeled were selected after measuring the duty cycles of 60 channels at GSM band. From these, three channels with different occupancy levels (high, medium and low), were chosen. Figure 1 presents results of power measure for three downlink channels during a week, with different power level. Spectrum analyzer configuration for this band was the following: a resolution bandwidth of $100 \mathrm{kHz}$ with a sweep time of $290 \mathrm{~ms}$, which guarantees GSM signal detection with a bandwidth of $200 \mathrm{kHz}$. Daily duty cycles from PUs at selected channels are shown in Figure 2. Threshold $(\lambda)$ used, which for this event is of $-89 \mathrm{dBm}$, was obtained from Equation (6) with a probability of false alarm (Pfa) of $1 \%$ [35]. $\lambda$ which is above of the detected noise floor of $-102 \mathrm{dBm}$ [1],

$$
P_{f a}=\frac{\Gamma\left(m, \frac{\lambda}{2}\right)}{\Gamma(m)}
$$


where $\Gamma($.$) y \Gamma(.,$.$) are complete and incomplete gamma functions, respectively, and m$ is the product of time times bandwidth.
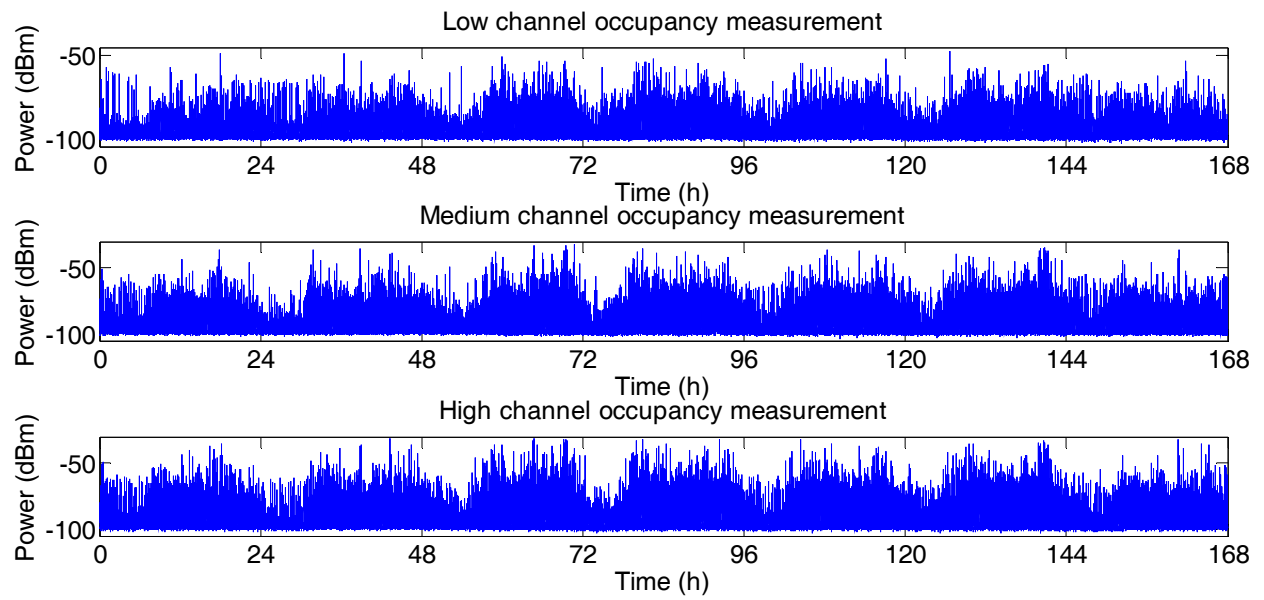

Figure 1. Power measurements for three global systems for mobile communications (GSM) band downlink channels.

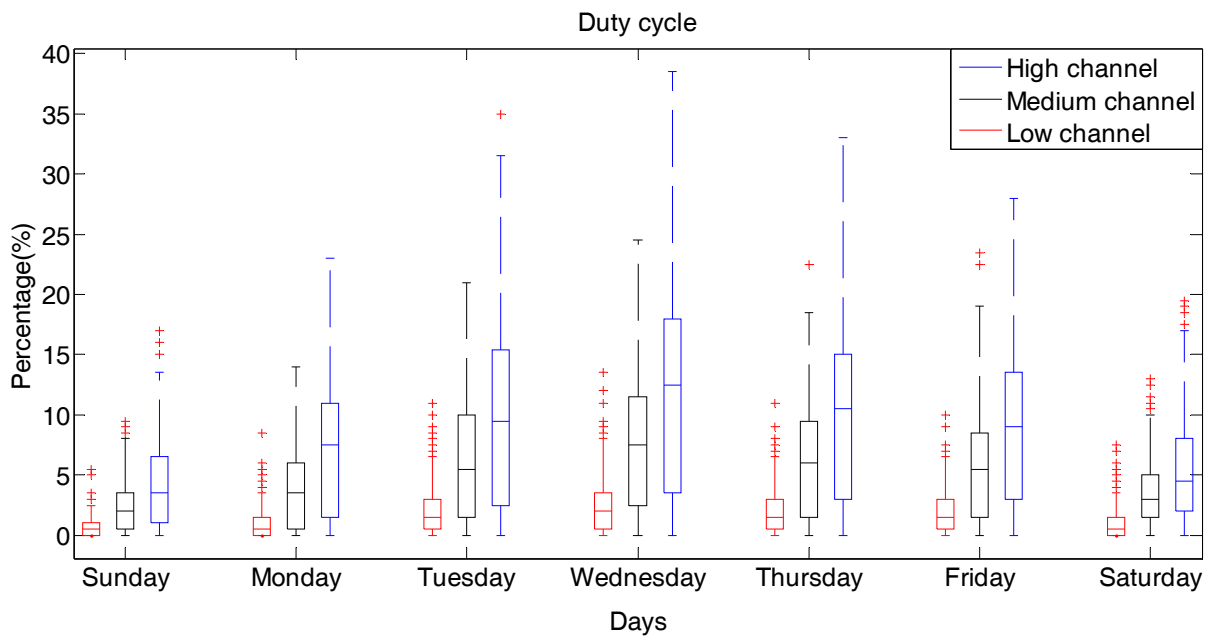

Figure 2. Duty cycles for three GSM band downlink channels.

In this work the HNM is calculated from the differences between measurements of received power performed outside at street level and indoors, in a building. These measurements were performed with a discone-type antenna and spectrum analyzer. The calculation allows analysis of the margin between a non-licensed device indoors, and a PU outdoors without interference due to shadowing. Table 1 shows the HNM found for each channel.

Table 1. Hidden node margin (HNM) for three different frequency channels for an urban environment.

\begin{tabular}{ccc}
\hline Low Channel HNM (dB) & Medium Channel HNM (dB) & High Channel HNM (dB) \\
\hline 9.2 & 11.9 & 6.8 \\
\hline
\end{tabular}

Figures 3-5 present histograms corresponding to opportunities distribution during time periods of GSM band channels; it is observed that such opportunities have an exponential behavior, whose approximate equations and the coefficient of determination $\left(R^{2}\right)$ are exhibited in each figure. Thus, the occurrence increases for the channel occupancy, especially for shorter time periods of use. For low, 
medium and high occupancy channels, total times of opportunities were approximately $84 \mathrm{~h}, 81 \mathrm{~h}$ and $78 \mathrm{~h}$, respectively, which indicates relatively low occupancy [2].

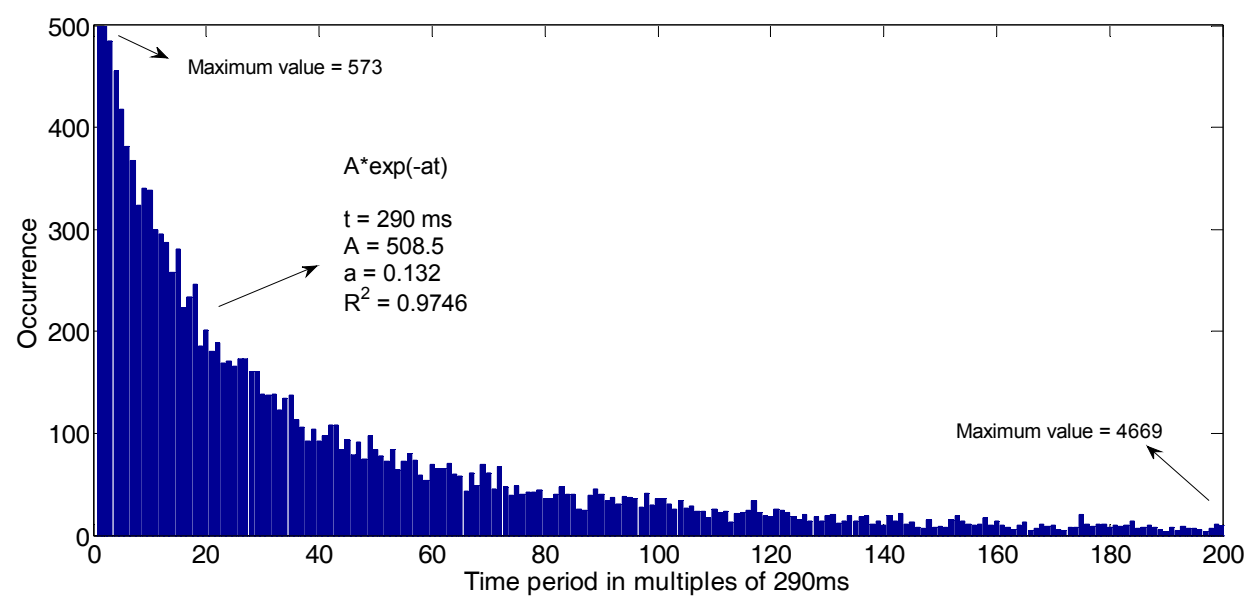

Figure 3. Time period opportunities distribution for low channel.

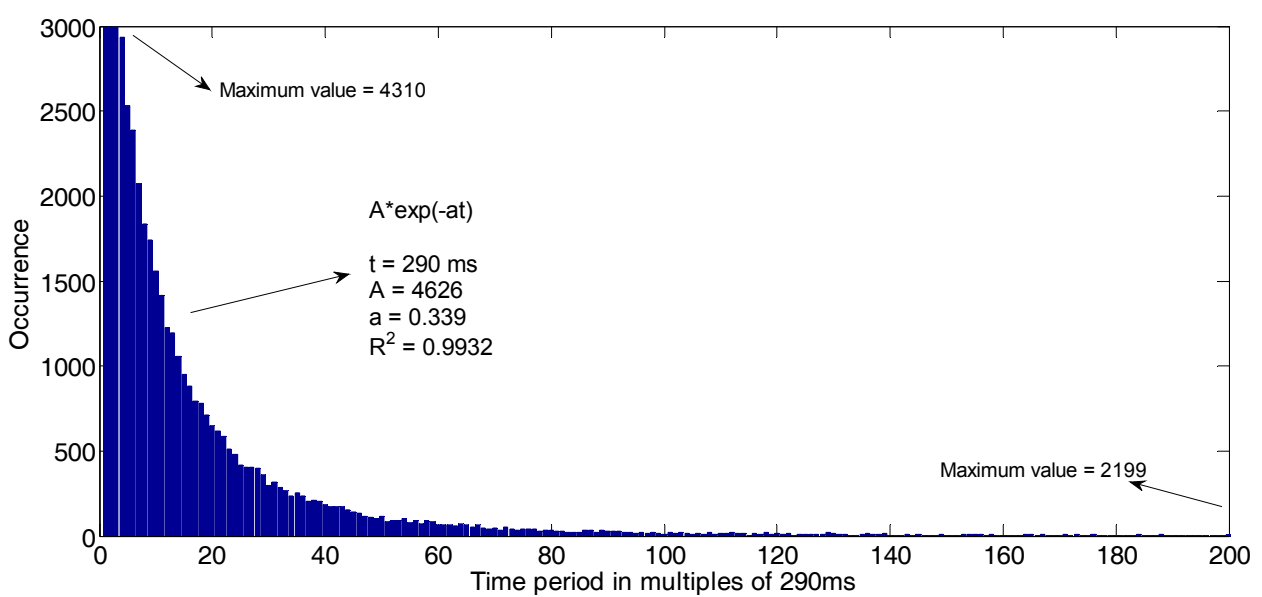

Figure 4. Time period opportunities distribution for medium channel.

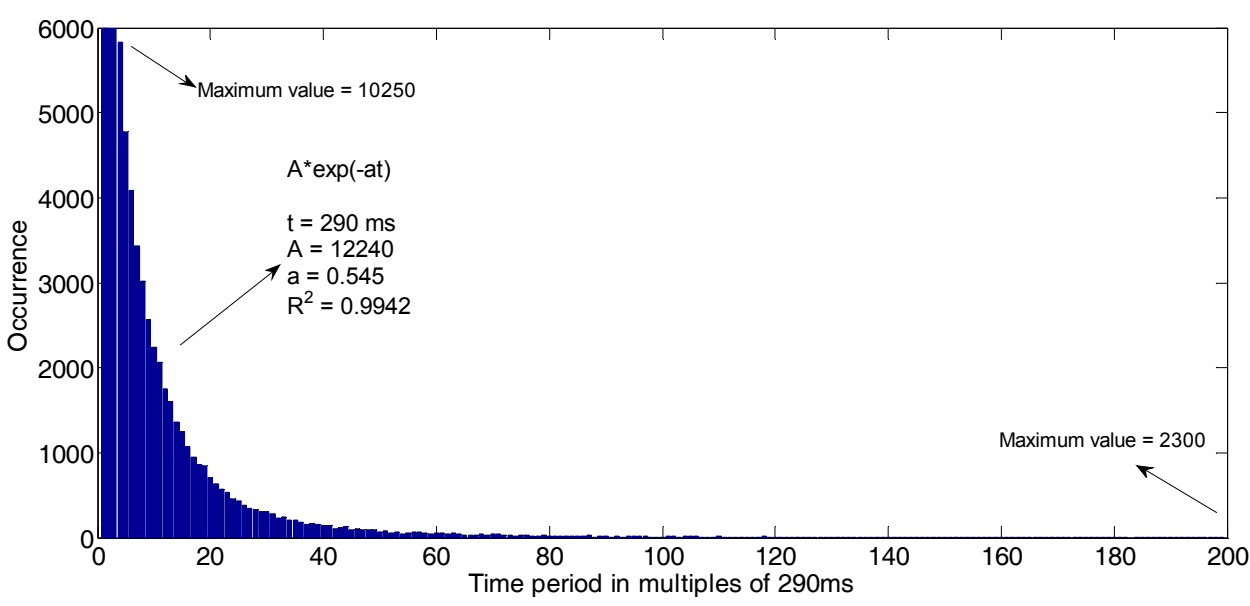

Figure 5. Time period opportunities distribution for high channel.

Following this, we proceeded to analyze the time series of measured channels over a week, which was equivalent to 1,062,514 samples. To do this, ACF is initially presented, as observed in Figure 6 . 
ACF diagrams for the three channels presents forms which are alternately positive and negative, decaying to zero, the values are in $95 \%$-confidence intervals, shown with the blue lines. Therefore, this indicates that there is correlation $[2,26]$.

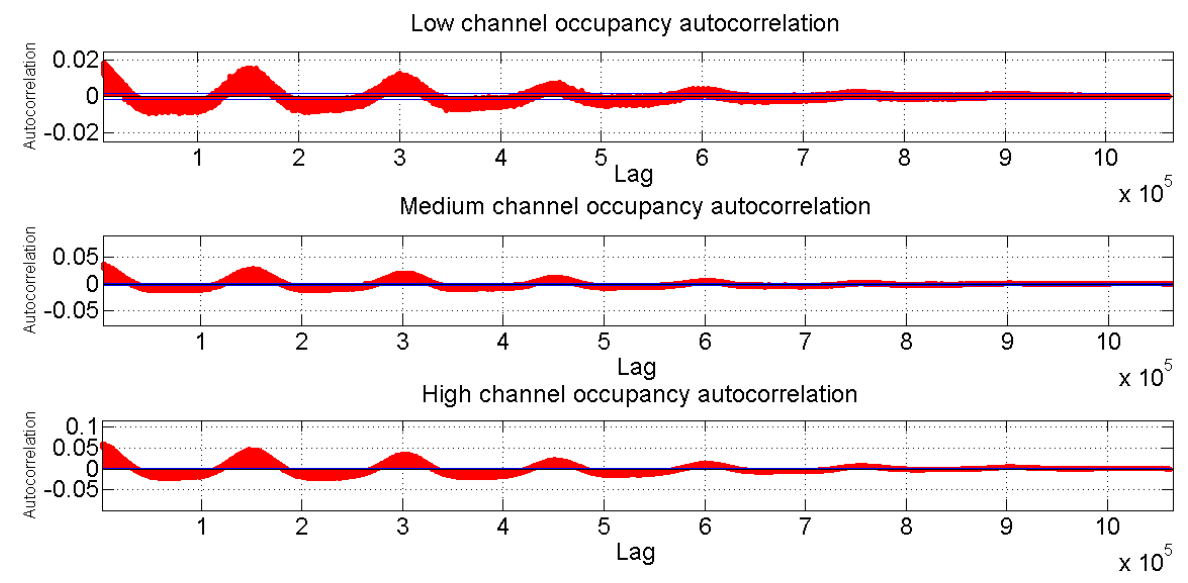

Figure 6. Autocorrelation for three GSM band downlink channels.

When analyzing channels stationarity of Figure 6, it is observed that the mean and variance are constant and similar to each other, on each one of the days from Monday to Friday. Therefore, measurements at the weekend are not taken into consideration when training the analyzed models, because the mean and variance are not similar and change in a significant way with regard to the measurements from Monday to Friday.

\subsection{Design of SARIMA Algorithm}

In Figure 7 the trend and seasonality are presented in occupancy level for the three channels. Seasonality had a period of $24 \mathrm{~h}$, practically without trend and with stationary components, which makes possible the use of a SARIMA model to forecast behavior of the GSM channels [26].
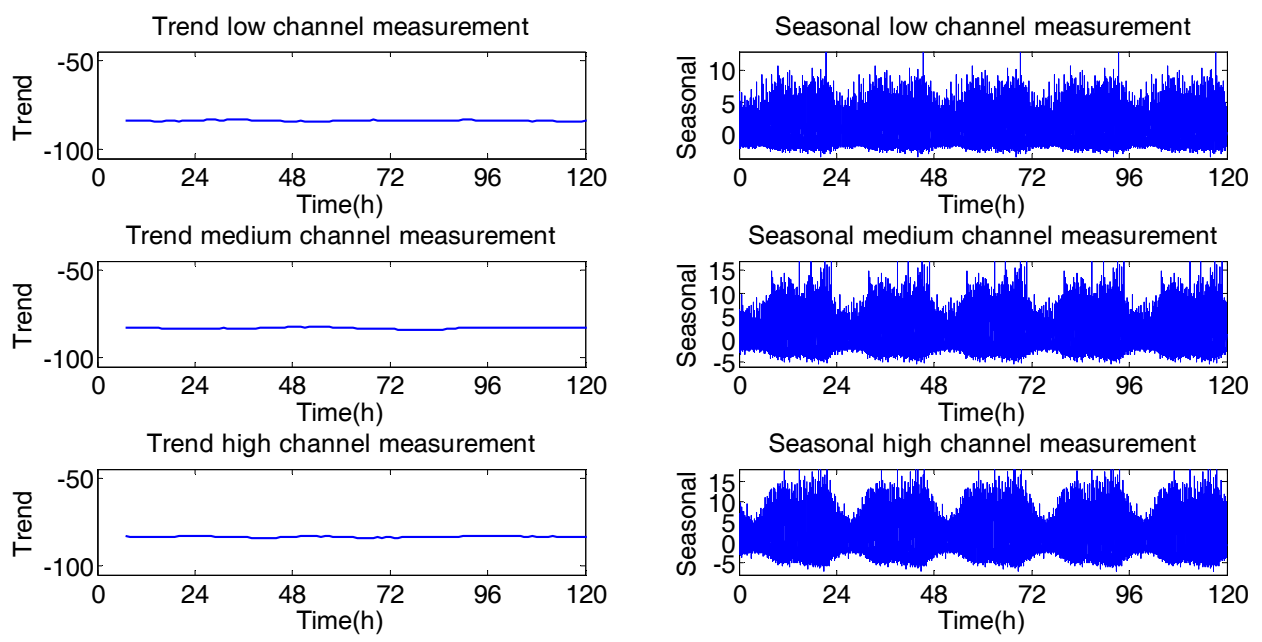

Figure 7. Seasonality and trend components of the GSM channels.

Delay difference $s$, which for this event is selected as five $\left(\Delta_{5}\right)$, was equivalent to the number of days of the week in which the signal was stationary [15]. Applying the augmented Dickey-Fuller test [36], in the series of three channels from Monday to Friday, the null hypothesis of unit root is rejected, which indicates stationarity. In order to find the parameters of SARIMA $(p, d, q)(P, D, Q) s$ model, $\mathrm{ACF}$ and PACF were calculated for $\Delta_{5}$ of respective channels, as shown in Figure 8. 

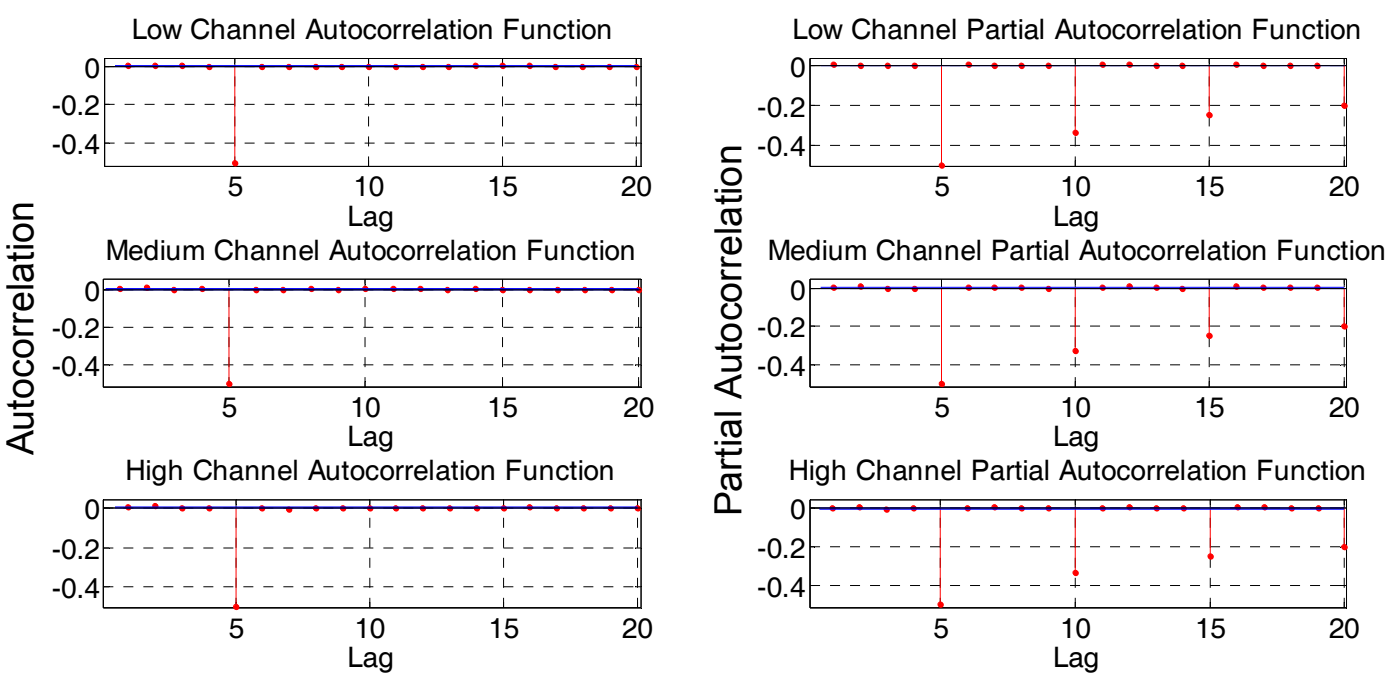

Figure 8. Simple and partial autocorrelation for GSM channels.

Using Box-Jenkins methodology [23], Figure 8 shows that PACF of $\Delta_{5}$ decays to zero with a seasonal pattern, and crosses confidence level initially in lag 5 for negative side. This suggests that a term non-seasonal AR(1) could be used, and a seasonal MA(5) could be added.

In order to avoid forecast overestimation (small variance and big errors), the Akaike information criterion (AIC) [37] was selected to evaluate different reasonable combinations, as is observed in Table 2 . Thus, selected models were: SARIMA $(1,0,5)(1,0,1)_{5}, \operatorname{SARIMA}(1,0,5)(0,0,1)_{5}$ and SARIMA $(1,0,5)(0,0,1)_{5}$, for occupancy levels of low, medium and high channels, respectively, and the characteristic equations, in the same order are:

$$
\begin{gathered}
(1-0.0135 B)\left(1-0.55 B^{5}\right)(1-B)\left(1-B^{5}\right) x_{t}=\left(1-0.997 B^{5}\right)\left(1-0.546 B^{5}\right) e_{t} \\
(1-0.0192 B)\left(1+0.996 B^{5}\right)(1-B)\left(1-B^{5}\right) x_{t}=\left(1+0.0085 B^{5}\right) e_{t} \\
(1-0.0199 B)\left(1-0.016 B^{5}\right)(1-B)\left(1-B^{5}\right) x_{t}=\left(1-0.994 B^{5}\right) e_{t}
\end{gathered}
$$

Table 2. Akaike information criterion (AIC) values for different seasonal autoregressive integrated moving average (SARIMA) models.

\begin{tabular}{ccccccccc}
\hline $\boldsymbol{p}$ & $\boldsymbol{d}$ & $\boldsymbol{q}$ & $\boldsymbol{P}$ & $\boldsymbol{D}$ & $\boldsymbol{Q}$ & $\begin{array}{c}\text { Low Occupancy } \\
\text { Channel AIC }\end{array}$ & $\begin{array}{c}\text { Medium Occupancy } \\
\text { Channel AIC }\end{array}$ & $\begin{array}{c}\text { High Occupancy } \\
\text { Channel AIC }\end{array}$ \\
\hline 1 & 0 & 5 & 0 & 0 & 1 & -8.24 & -30.6 & -50.82 \\
1 & 0 & 5 & 1 & 0 & 0 & -8.3 & -32.7 & -51.7 \\
1 & 1 & 5 & 0 & 0 & 1 & -14.1 & -46.9 & -76.2 \\
1 & 0 & 5 & 1 & 0 & 1 & -8.19 & -32.6 & -50.9 \\
\hline
\end{tabular}

\subsection{Design of GARCH Algorithm}

When analyzing in detail the large amount of acquired information, the existence of standard deviation was observed; therefore the GARCH algorithm was used to forecast the behavior of measured series. Stochastic models ARIMA and SARIMA are methods for univariate modeling. The main difference among former models and GARCH model lies in the constant variance assumption.

Even though for the developed algorithm there is stationarity in original signal from Monday to Friday, for this case the fifth difference is developed because there is a greater degree of stationarity. 
In Figure 9 the difference for each channel is presented. Channel measurements are converted into returns by logarithmic transformation. The logarithmic returns are defined in Equation (10),

$$
r_{t}=\ln \frac{P_{t}}{P_{t-1}}
$$

where $P_{t}$ is power value in time $t$ and $P_{t-1}$ is power value in time ${ }_{t-1}$.
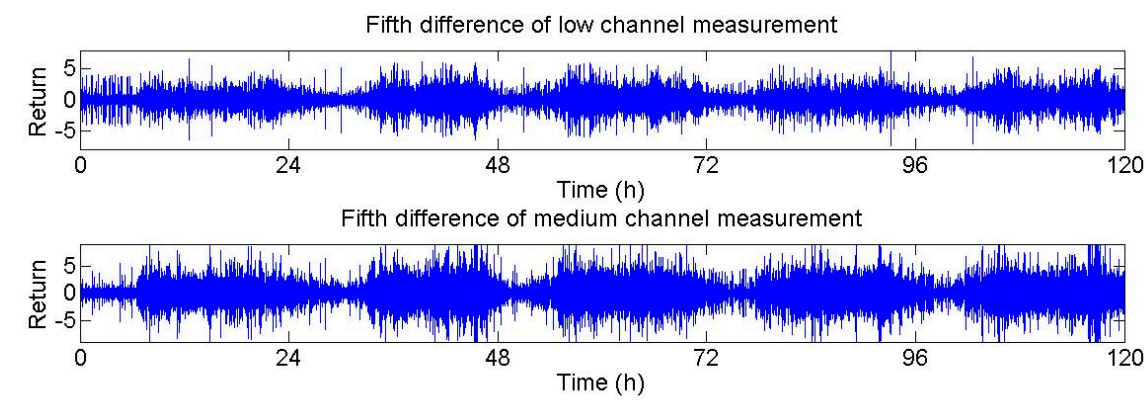

Fifth difference of high channel measurement

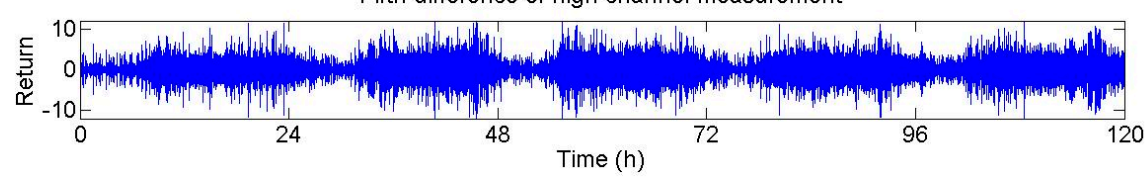

Figure 9. Fifth difference of measured powers in channels of GSM band.

Now we present a formal statistical test in order to establish the presence of ARCH effects in the data and correlation. $\mathrm{H}=0$ implies that there exist no significant correlation as well as $\mathrm{H}=1$ indicates that there exists a significant correlation. In Tables 3 and 4, all the $p$ values show that Ljung-Box-Pierce Q-Test and Engle ARCH Test in lag 10, 15 and 20 are significant, revealing the presence of ARCH effects (heteroskedasticity), indicating that GARCH modeling is appropriate.

Table 3. Ljung-Box-Pierce Q-Test for autocorrelation: (at 95\% confidence) for GSM channels.

\begin{tabular}{ccccccc}
\hline Lag & H & $\boldsymbol{p}$ Value & $\begin{array}{c}\text { Low Channel } \\
\text { Statistical Test }\end{array}$ & $\begin{array}{c}\text { Medium Channel } \\
\text { Statistical Test }\end{array}$ & $\begin{array}{c}\text { High Channel } \\
\text { Statistical Test }\end{array}$ & $\begin{array}{c}\text { Critical } \\
\text { Value }\end{array}$ \\
\hline 10 & 1 & 0 & 725,124 & 731,923 & 731,240 & 18.3 \\
15 & 1 & 0 & 725,136 & 731,956 & 731,266 & 24.99 \\
20 & 1 & 0 & 725,138 & 731,996 & 731,313 & 31.41 \\
\hline
\end{tabular}

Table 4. Engle autoregressive conditional heteroskedasticity ( $\mathrm{ARCH}$ ) test: (at 95\% confidence) for GSM channels.

\begin{tabular}{ccccccc}
\hline Lag & H & $\boldsymbol{p}$ Value & $\begin{array}{c}\text { Low Channel } \\
\text { Statistical Test }\end{array}$ & $\begin{array}{c}\text { Medium Channel } \\
\text { Statistical Test }\end{array}$ & $\begin{array}{c}\text { High Channel } \\
\text { Statistical Test }\end{array}$ & $\begin{array}{c}\text { Critical } \\
\text { Value }\end{array}$ \\
\hline 10 & 1 & 0 & 574,940 & 578,554 & 576,595 & 18.3 \\
15 & 1 & 0 & 578,008 & 581,225 & 579,079 & 24.99 \\
20 & 1 & 0 & 578,710 & 581,829 & 579,500 & 31.41 \\
\hline
\end{tabular}

Dependence in data $x_{1}, \ldots, x_{n}$ was determined by computing correlations. This was done by representing the ACF.

If the time series is the result of a completely random phenomenon, the autocorrelation should be close to zero for all time-lag separations. Otherwise, one or more of the autocorrelations will be significantly different from zero. Another useful way to examine dependencies of the series is to revise the PACF, where the dependence of intermediate elements (those within the lag) is eliminated. 
In Figure 10, graphs of ACF, PACF and ACF of square returns present the existence of correlation in data of channel occupancy.
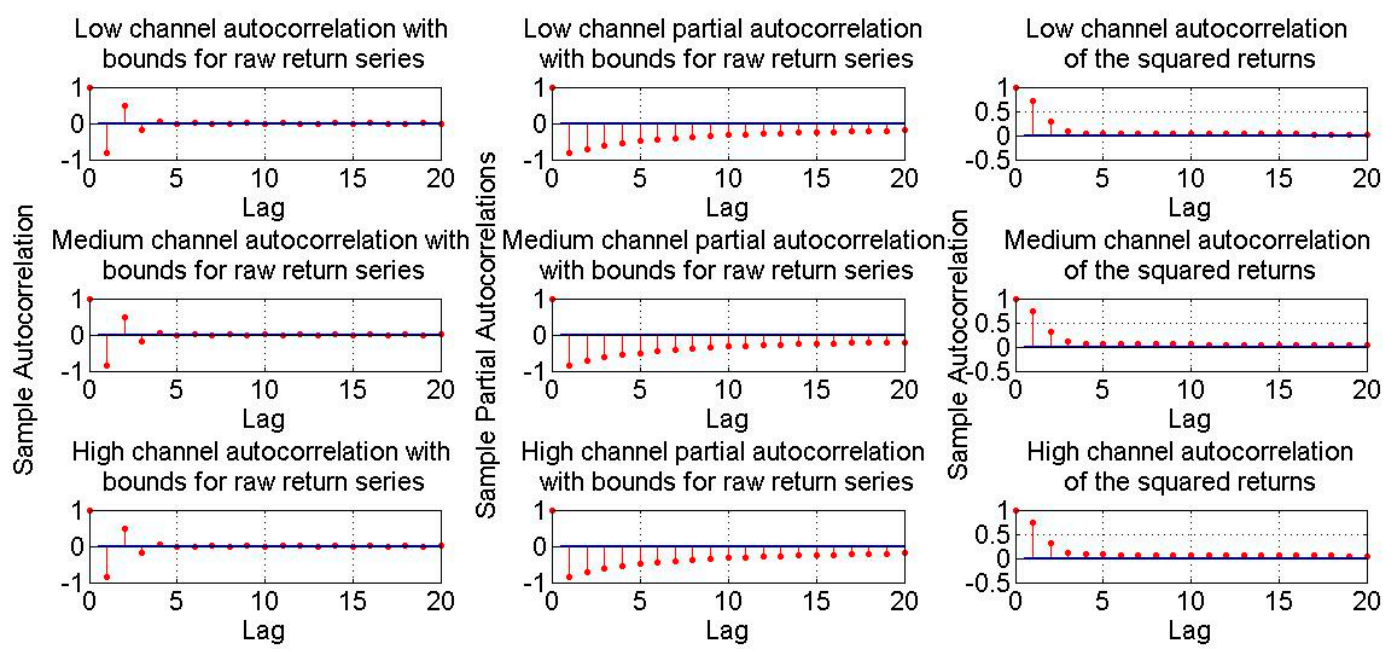

Figure 10. Correlation graphs for GSM band channels.

Below, in Tables 5-7, the evaluation and selection of the GARCH model for each channel was performed.

Table 5. Generalized autoregressive conditional heteroskedasticity (GARCH) model comparison for low channel. SMAPE: symmetrical mean absolute percentage error; MAPE: mean absolute percentage error; MAE: mean absolute error.

\begin{tabular}{cccccccc}
\hline Model & AIC & $\begin{array}{c}\text { Bayesian } \\
\text { Information } \\
\text { Criterion (BIC) }\end{array}$ & $\begin{array}{c}\text { Standard } \\
\text { Error }\end{array}$ & $\begin{array}{c}\text { Log } \\
\text { Likelihood }\end{array}$ & SMAPE & MAPE & MAE \\
\hline GARCH(0,1) & 201,838 & 201,873 & $7.8 \times 10^{-4}$ & $96,127.5$ & -0.0249 & 0.0253 & 2.3606 \\
GARCH(1,1) & 192,263 & 192,309 & $7.82 \times 10^{-4}$ & $96,127.5$ & -0.0249 & 0.0253 & 2.3604 \\
GARCH(0,2) & 192,622 & 192,649 & $7.8 \times 10^{-4}$ & $96,127.5$ & -0.0248 & 0.0252 & 2.3492 \\
GARCH(1,2) & 192,265 & 192,299 & 0.0016 & $96,127.5$ & -0.0244 & 0.0248 & 2.3075 \\
GARCH(2,1) & 191,587 & 191,621 & $7.33 \times 10^{-4}$ & $96,127.5$ & -0.0251 & 0.0255 & 2.3792 \\
GARCH(2,2) & 191,581 & 191,622 & 0.0034 & $96,127.5$ & -0.0243 & 0.0247 & 2.3060 \\
\hline
\end{tabular}

Table 6. GARCH model comparison for medium channel.

\begin{tabular}{cccccccc}
\hline Model & AIC & BIC & Standard Error & Log Likelihood & SMAPE & MAPE & MAE \\
\hline GARCH(0,1) & 876,834 & 876,854 & $7.6 \times 10^{-4}$ & 422,041 & -0.0374 & 0.0393 & 3.4198 \\
GARCH(1,1) & 844,089 & 844,117 & $6.6 \times 10^{-4}$ & 422,041 & -0.0427 & 0.0440 & 3.8676 \\
GARCH(0,2) & 844,984 & 845,012 & $6.6 \times 10^{-4}$ & 422,041 & -0.0375 & 0.0395 & 3.4385 \\
GARCH(1,2) & 844,091 & 844,125 & 0.0012 & 422,041 & -0.0411 & 0.0429 & 3.7699 \\
GARCH(2,1) & 843,470 & 843,504 & $6.0 \times 10^{-4}$ & 422,041 & -0.0410 & 0.0427 & 3.7531 \\
GARCH(2,2) & 843,472 & 843,513 & $5.0 \times 10^{-4}$ & 422,041 & -0.0434 & 0.0452 & 3.9895 \\
\hline
\end{tabular}

Table 7. GARCH model comparison for high channel.

\begin{tabular}{cccccccc}
\hline Model & AIC & BIC & Standard Error & Log Likelihood & SMAPE & MAPE & MAE \\
\hline GARCH(0,1) & $1,223,114$ & $1,223,135$ & $7.8 \times 10^{-4}$ & 608,609 & -0.0514 & 0.0542 & 4.6565 \\
GARCH $(1,1)$ & $1,217,225$ & $1,217,252$ & $6.6 \times 10^{-4}$ & 608,609 & -0.0551 & 0.0580 & 5.0138 \\
GARCH(0,2) & $1,220,306$ & $1,220,333$ & $6.7 \times 10^{-4}$ & 608,609 & -0.0534 & 0.0557 & 4.7957 \\
GARCH $(1,2)$ & $1,217,227$ & $1,217,261$ & $5.3 \times 10^{-4}$ & 608,609 & -0.0566 & 0.0591 & 5.1279 \\
GARCH $(2,1)$ & $1,214,308$ & $1,214,343$ & $6.5 \times 10^{-4}$ & 608,609 & -0.0540 & 0.0570 & 4.9224 \\
GARCH $(2,2)$ & $1,214,310$ & $1,214,352$ & $5.4 \times 10^{-4}$ & 608,609 & -0.0620 & 0.0675 & 5.9397 \\
\hline
\end{tabular}


The GARCH model selection for each channel was done by fulfilling $\alpha_{i}+\beta_{i}<1$ criterion, so the model is stationary, and then taking into account the more proximate values to zero of MAE, MAPE and SMAPE from Tables 5-7. Therefore, the selected models for low, medium and high channel are $\operatorname{GARCH}(2,2), \operatorname{GARCH}(0,2)$ and $\operatorname{GARCH}(0,1)$, respectively.

Parameters for low channel model were estimated and are presented in Table 8. GARCH$(2,2)$, where $\alpha_{1}+\alpha_{2}+\beta_{1}+\beta_{2}<1$ is fulfilled.

Table 8. Parameters estimation for low channel model.

\begin{tabular}{cccc}
\hline Parameter & Estimated Value & Standard Error & $\boldsymbol{t}$ Value \\
\hline$\mu$ & -96.112 & 0.0019308 & $-49,778.3308$ \\
$\alpha_{0}$ & 0.003516 & 0.00041447 & 8.4833 \\
GARCH(1) & 0.098255 & 0.19212 & 0.5114 \\
GARCH(2) & 0.90062 & 0.19201 & 4.6905 \\
ARCH(1) & 0.00029573 & 0.00018772 & 1.5753 \\
ARCH(2) & 0 & 0.00020886 & 0 \\
\hline
\end{tabular}

Thus, the model according to Table 8 is presented in Equations (11) and (12),

$$
\begin{gathered}
r_{t}=-96.112+\epsilon_{t} \\
\sigma_{t}^{2}=0.003516+0.098255 \sigma_{t-1}^{2}+0.90062 \sigma_{t-2}^{2}+0.00029573 \epsilon_{t-1}^{2}
\end{gathered}
$$

For medium channel, GARCH $(0,2)$, model values presented in Table 9 are estimated.

Table 9. Parameters estimation for medium channel model.

\begin{tabular}{cccc}
\hline Parameter & Estimated Value & Standard Error & $\boldsymbol{t}$ Value \\
\hline$\mu$ & -95.061 & 0.0024331 & $-39,069.8019$ \\
$\alpha_{0}$ & 5 & 0.012924 & 386.8834 \\
$\mathrm{ARCH}(1)$ & 0.085692 & 0.0010392 & 82.4572 \\
$\mathrm{ARCH}(2)$ & 0.088298 & 0.0010582 & 83.4378 \\
\hline
\end{tabular}

Therefore, Equations (13) and (14) are obtained,

$$
\begin{gathered}
r_{t}=-95.061+\epsilon_{t} \\
\sigma_{t}^{2}=5+0.085692 \epsilon_{t-1}^{2}+0.088298 \epsilon_{t-2}^{2}
\end{gathered}
$$

For high channel, GARCH$(0,1)$, the following parameters were obtained, as shown in Table 10.

Table 10. Parameters estimation for high channel model.

\begin{tabular}{cccc}
\hline Parameter & Estimated Value & Standard Error & $\boldsymbol{t}$ Value \\
\hline$\mu$ & -94.585 & 0.0026236 & $-36,051.8702$ \\
$\alpha_{0}$ & 5 & 0.015341 & 325.9324 \\
$\mathrm{ARCH}(1)$ & 0.86058 & 0.0044771 & 192.2169 \\
\hline
\end{tabular}

Then the model is described in Equations (15) and (16),

$$
\begin{gathered}
r_{t}=-94.585+\epsilon_{t} \\
\sigma_{t}^{2}=5+0.86058 \epsilon_{t-1}^{2}
\end{gathered}
$$


ARCH-GARCH model analysis is based on evaluation of standardized residuals [31]. One assumption with GARCH model is that for a good model, residuals should follow a white noise process. This is to say that it is expected that residuals be at random, independent and identically distributed, following a normal distribution. Figure 11 presents the relationship between innovations (residuals) derivate from adjusted model, the corresponding conditional standard deviations and returns. Figure 11 shows that both innovations and returns exhibit variations. In the following we intend to find out if by performing GARCH the autocorrelation of the standardized innovations disappears, which would indicate the effectiveness of GARCH model.

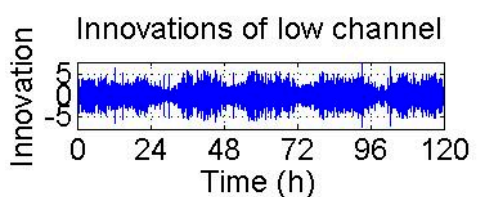

Conditional standard deviations

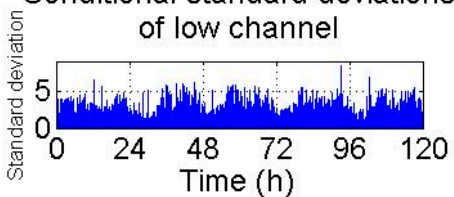

Returns of low channel

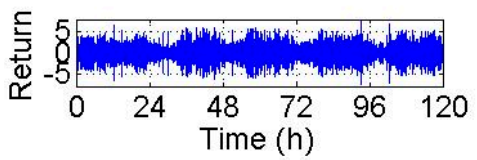

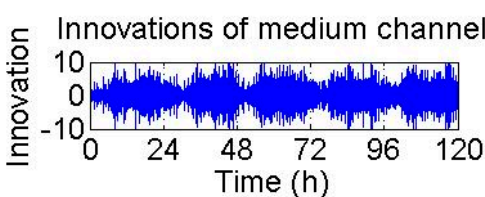

Conditional standard deviations

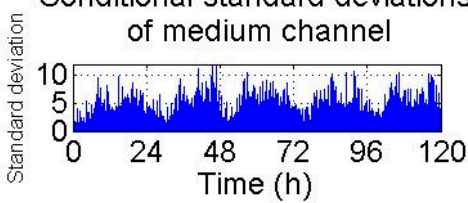

Returns of medium channel

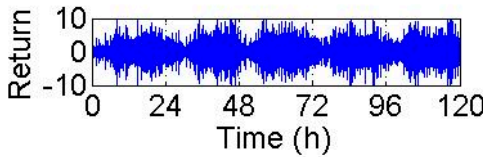

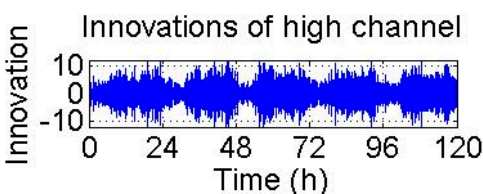

Conditional standard deviations

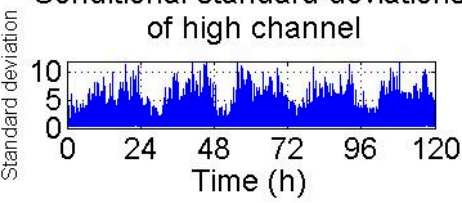

Returns of high channel

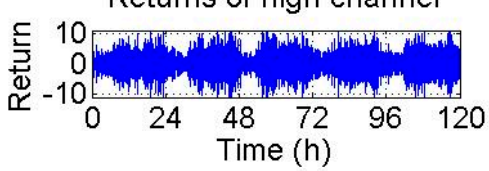

Figure 11. Innovations, conditional standard deviations and returns of GSM channels.

Figure 12 corresponds to the autocorrelation of the squared standardized innovations, in which correlation was not observed.

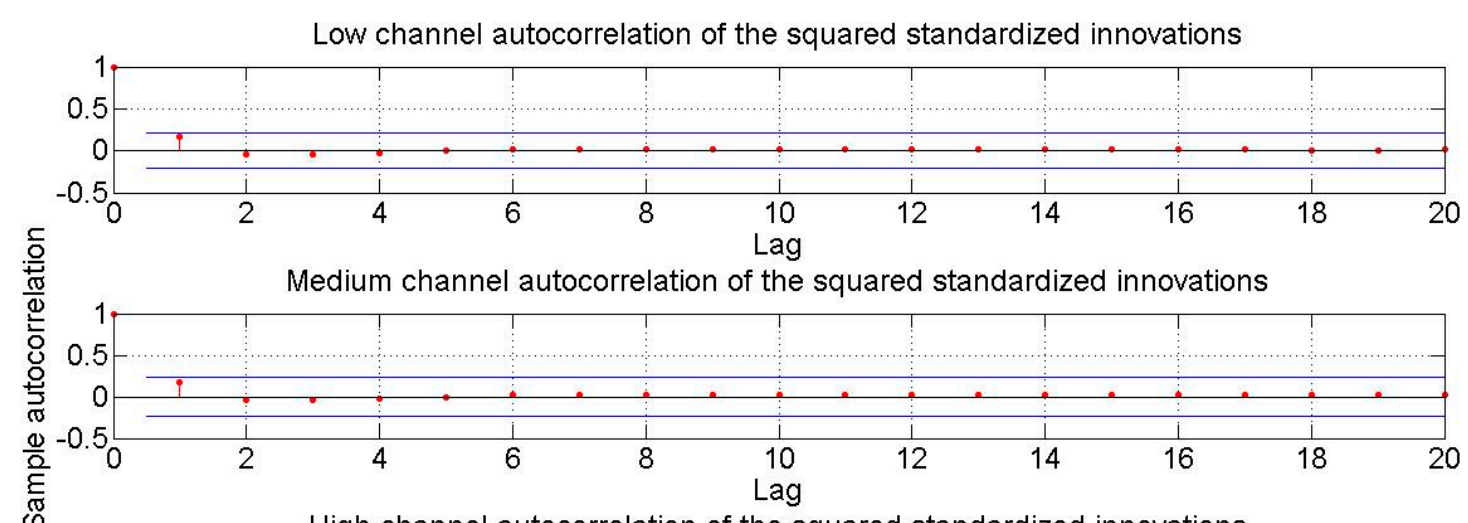

High channel autocorrelation of the squared standardized innovations

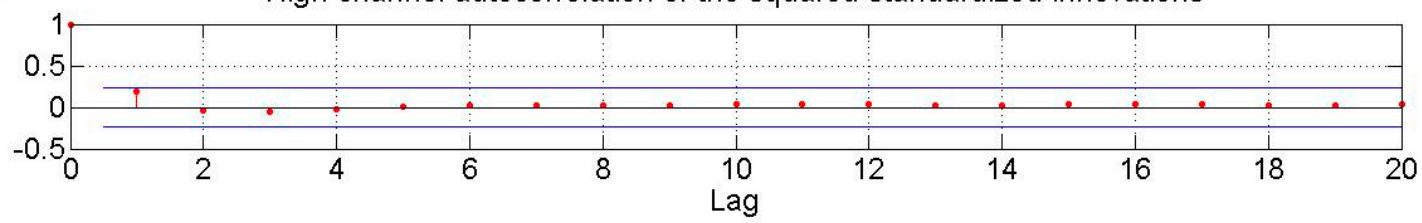

Figure 12. Autocorrelation of the squared standardized innovations of GSM channels.

In Tables 11 and 12, results of Ljung-Box-Pierce Q-Test and Engle ARCH test for later analysis are presented using standardized innovations. These tests indicate no presence of correlation or ARCH effects. We have GARCH effects and also correlation between innovations that disappear after 
treating the data. Therefore, the GARCH model is a proper model for explaining the variances of the three channels.

Table 11. Ljung-Box-Pierce Q-Test in standardized innovations for GSM channels.

\begin{tabular}{ccccccccc}
\hline Lag & $\mathbf{H}$ & $\begin{array}{c}\text { Low } \\
\text { Channel } \\
\boldsymbol{p} \text { Value }\end{array}$ & $\begin{array}{c}\text { Medium } \\
\text { Channel } \\
\boldsymbol{p} \text { Value }\end{array}$ & $\begin{array}{c}\text { High } \\
\text { Channel } \\
\boldsymbol{p} \text { Value }\end{array}$ & $\begin{array}{c}\text { Low Channel } \\
\text { Statistical Test }\end{array}$ & $\begin{array}{c}\text { Medium Channel } \\
\text { Statistical Test }\end{array}$ & $\begin{array}{c}\text { High Channel } \\
\text { Statistical Test }\end{array}$ & $\begin{array}{c}\text { Critical } \\
\text { Value }\end{array}$ \\
\hline 10 & 0 & 0.424 & 0.402 & 0.701 & 25,787 & 26,701 & 33,455 \\
15 & 0 & 0.7014 & 0.6883 & 0.8236 & 26,447 & 28,617 & 37,143 \\
20 & 0 & 0.947 & 0.876 & 0.9355 & 26,945 & 30,313 & 24.99 \\
\hline
\end{tabular}

Table 12. Engle ARCH test in standardized innovations for GSM channels.

\begin{tabular}{ccccccccc}
\hline Lag & $\mathbf{H}$ & $\begin{array}{c}\text { Low } \\
\text { Channel } \\
\boldsymbol{p} \text { Value }\end{array}$ & $\begin{array}{c}\text { Medium } \\
\text { Channel } \\
\boldsymbol{p} \text { Value }\end{array}$ & $\begin{array}{c}\text { High } \\
\text { Channel } \\
\boldsymbol{p} \text { Value }\end{array}$ & $\begin{array}{c}\text { Low Channel } \\
\text { Statistical Test }\end{array}$ & $\begin{array}{c}\text { Medium Channel } \\
\text { Statistical Test }\end{array}$ & $\begin{array}{c}\text { High Channel } \\
\text { Statistical Test }\end{array}$ & $\begin{array}{c}\text { Critical } \\
\text { Value }\end{array}$ \\
\hline 10 & 0 & 0.539 & 0.479 & 0.6212 & 26,930 & 27,093 & 33,757 & 18.3 \\
15 & 0 & 0.776 & 0.7144 & 0.7697 & 27,432 & 28,443 & 36,248 & 24.99 \\
20 & 0 & 0.908 & 0.863 & 0.8841 & 27,792 & 29,443 & 38,240 & 31.41 \\
\hline
\end{tabular}

Normality verification was performed by analyzing histograms of residuals and normal probability graph, as shown in Figure 13. The histograms of the three channels shows that the residuals are normally distributed. In turn, the probability graph confirms that residuals respond to a normal distribution, since most of data are spread along the straight line.
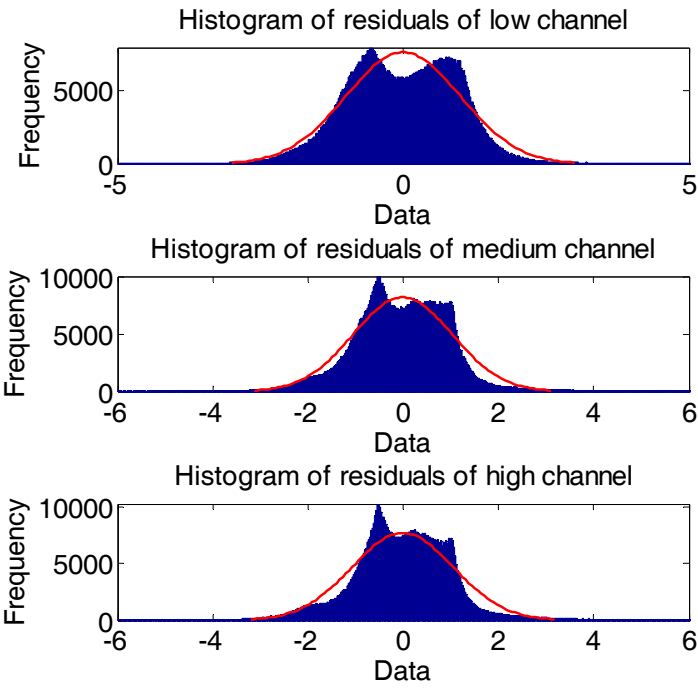

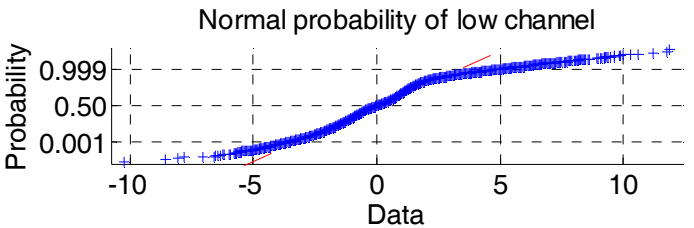

Normal probability of medium channel

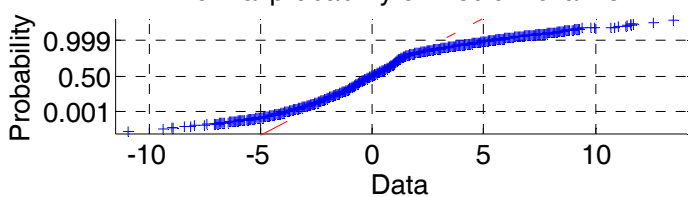

Normal probability of high channel

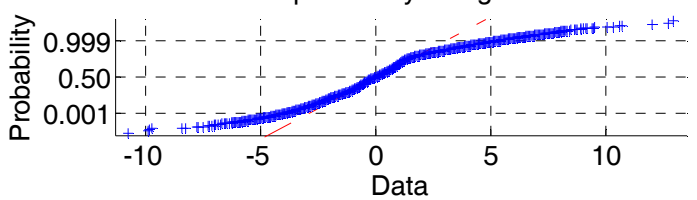

Figure 13. Histogram of residuals and normal probability for GSM channels.

\section{Results and Discussion}

In Figure 14 an example is displayed where there is application of the designed time series algorithms. Here, the interaction between the CR user and the primary base station (BS) is shown by received power from the primary transmitter. This is represented by the oval and the direction of the arrows. The CR user can forecast the power level it will receive from the primary BS.

In the example of Figure 14, in order to analyze the SARIMA and GARCH algorithms, the forecast of the power is performed by the $\mathrm{CR}$ user, making a comparison with the spectrum analyzer in which the measurements were made. However, this depends on the architecture of CR deployed in the environment. Due to the processor and power consumption being more limited in the CR user's computer, use of infrastructure architecture is recommended, where the forecast is carried out by the 
CR BS. This provides a better processor than that of the CR user, and has no limitations on power consumption. However, there is a time period between data capture in the environment and the processing, which adds a delay to the response. This must not be ignored, but the forecast helps to reduce the negative impact of the delayed response.

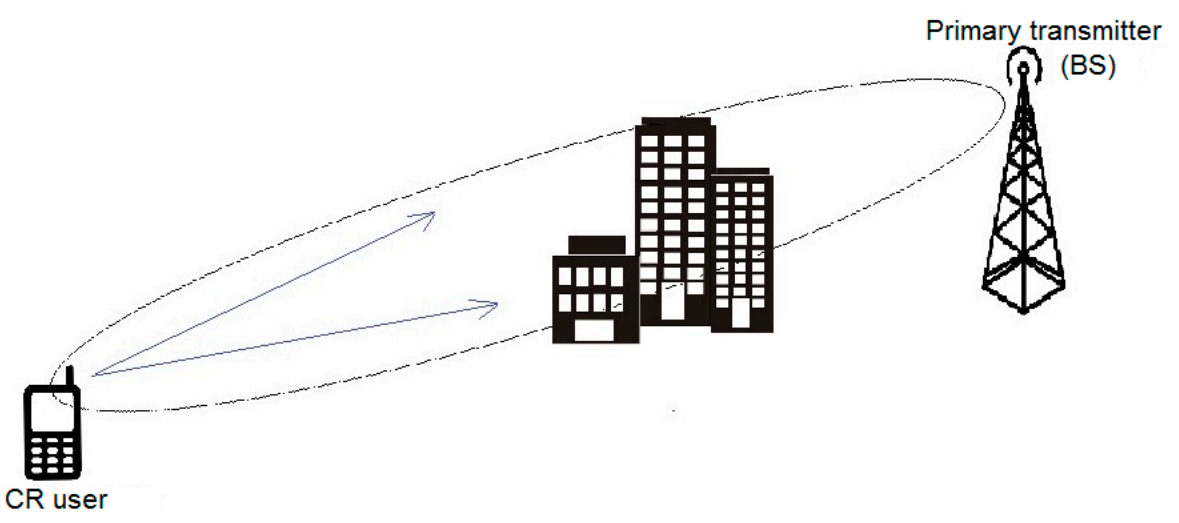

Figure 14. Example of application to forecast the received power from the base station (BS). CR: cognitive radio.

The analysis of the precision of the forecast made with the SARIMA and GARCH algorithms is presented, as follows. Figure 15 shows the SARIMA algorithm forecasts obtained from Equations (7)-(9), and from GARCH algorithm based on Equations (11)-(16). This was contrasted with the power measured data of Friday from 5:00 p.m. to 6:00 p.m. This period was chosen since during this time an increased use of the channels by PUs was perceived.
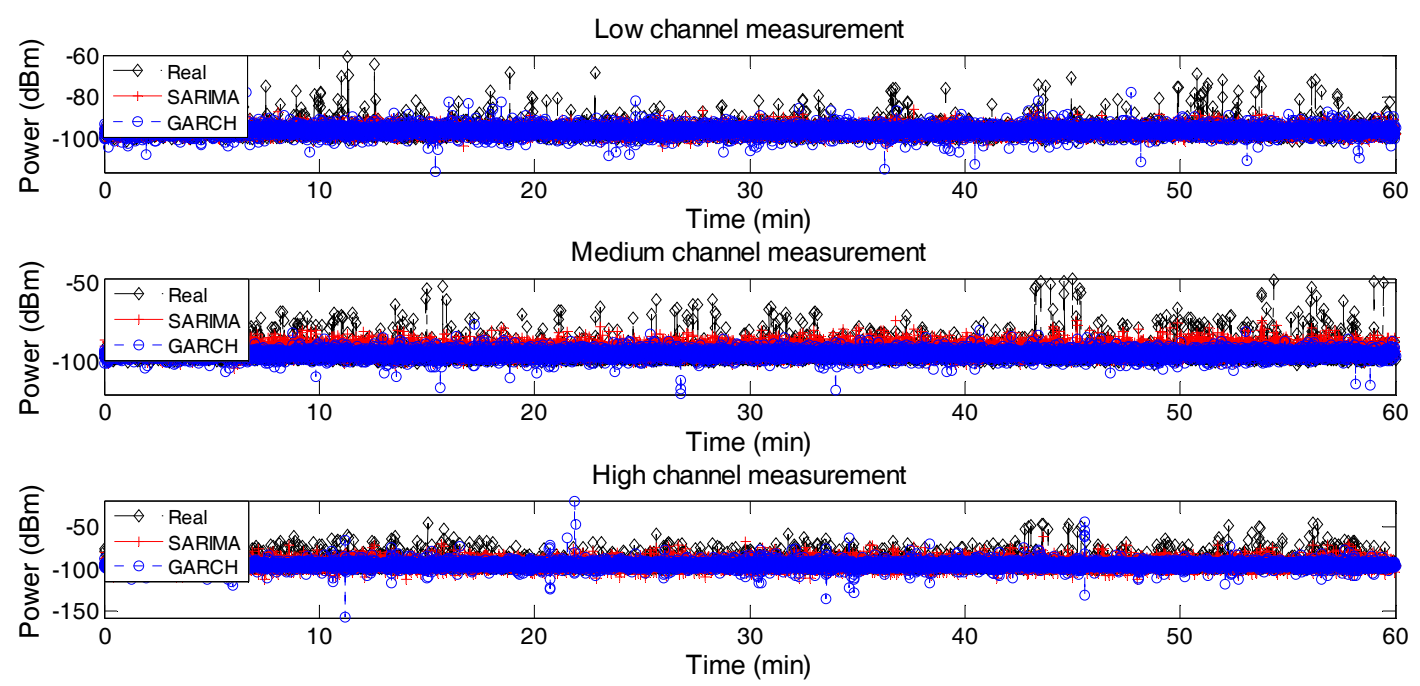

Figure 15. GSM channel series and forecast series with SARIMA and GARCH algorithms.

In Figures 16 and 17, availability and occupancy times of the measured and forecast channels are presented. Availability time allows us to analyze the precision with which SUs could use availability time in GSM channels for a CR system. In the same way, occupancy time examines the precision during time in which PUs use GSM channels. Average precisions obtained between actual and forecast data for availability times were: $82 \%, 54 \%$ and $60 \%$, for the SARIMA algorithm; and $31 \%, 30 \%$ and $43 \%$, for the GARCH algorithm, corresponding to channels with low, medium and high occupation, respectively. Average precisions for occupation times between real data and forecast data are equivalent to $58 \%$, $77 \%$ and $78 \%$ for the SARIMA algorithm; and $44 \%, 46.6 \%$ and $44.2 \%$, for the GARCH algorithm, 
corresponding to channels with low, medium and high occupancy levels, respectively. Additionally, as expected, for each algorithm there is an inversely proportional relationship between channel occupancy and availability time, as well as a directly proportional relationship between occupancy probability and occupancy time of the channels.

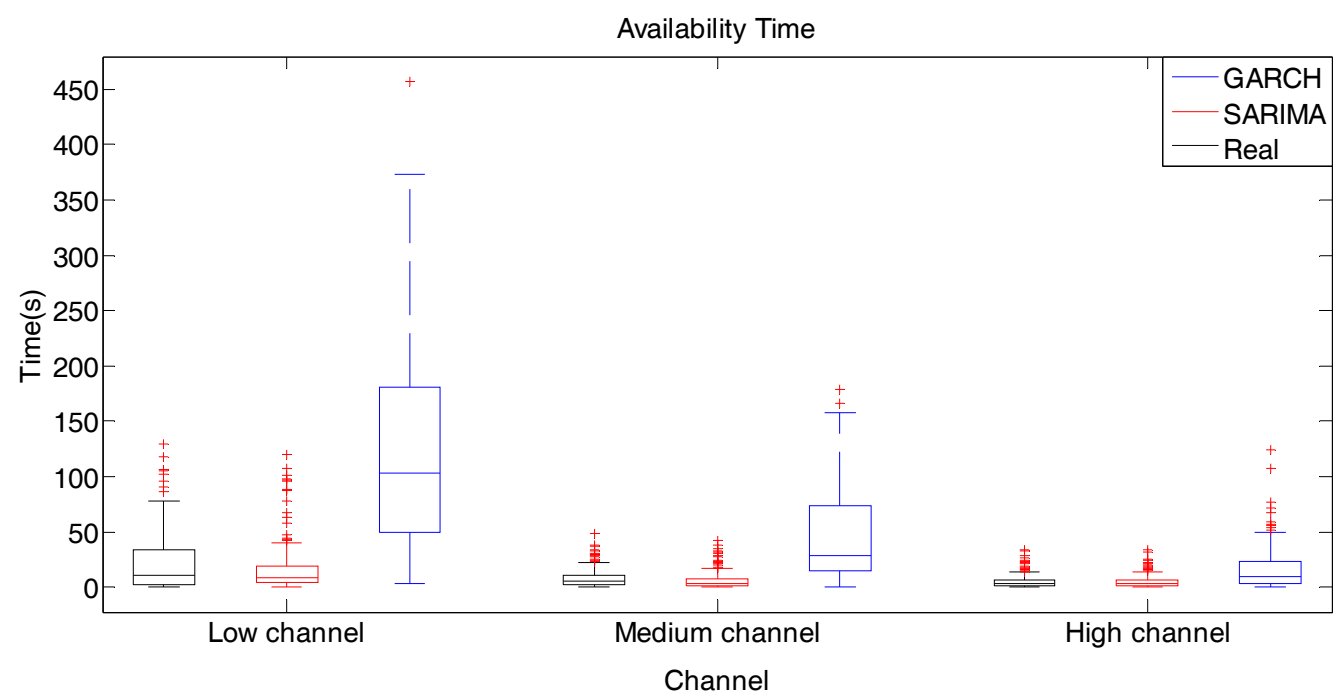

Figure 16. Availability time of forecasted channels.

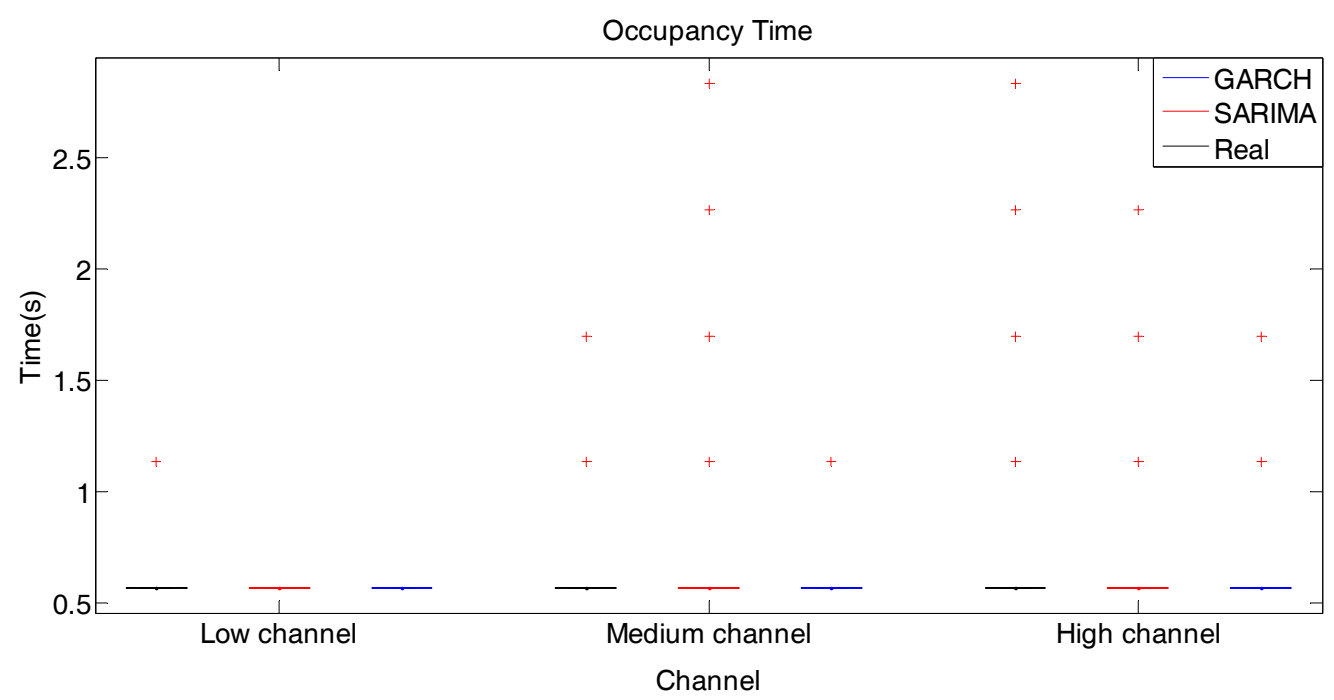

Figure 17. Occupancy time of forecasted channels.

In Table 13 forecast and measured data are compared to different methods for error estimation such as SMAPE, MAPE and MAE. Results of Table 13 point that only in data forecast for the medium occupancy channel, the GARCH algorithm presents smaller errors than the SARIMA algorithm.

Table 13. Error variables comparison for forecasted values.

\begin{tabular}{|c|c|c|c|c|c|c|}
\hline $\begin{array}{c}\text { Channel } \\
\text { Occupancy }\end{array}$ & $\begin{array}{l}\text { SMAPE- } \\
\text { SARIMA }\end{array}$ & $\begin{array}{l}\text { SMAPE- } \\
\text { GARCH }\end{array}$ & $\begin{array}{l}\text { MAPE- } \\
\text { SARIMA }\end{array}$ & $\begin{array}{l}\text { MAPE- } \\
\text { GARCH }\end{array}$ & $\begin{array}{c}\text { MAE- } \\
\text { SARIMA }\end{array}$ & $\begin{array}{c}\text { MAE- } \\
\text { GARCH }\end{array}$ \\
\hline Low & -0.0170 & -0.0243 & 0.0172 & 0.0247 & 1.6042 & 2.306 \\
\hline Medium & -0.0470 & -0.0375 & 0.0466 & 0.0395 & 4.2987 & 3.4385 \\
\hline High & -0.0488 & -0.0514 & 0.0497 & 0.0542 & 4.4195 & 4.6565 \\
\hline
\end{tabular}


Comparison of performance in forecast is shown in Figure 18, developed on a computer with dual-core processor $2.4 \mathrm{GHz}$ and $4 \mathrm{~GB}$ of random access memory (RAM) memory. Here is observed that in general, for each model, the higher the observation time the lower the prediction error. However, this entails no significance. Prediction error is lower in forecast of GARCH algorithms, but a longer observation time in connection to SARIMA algorithm forecast is still necessary. Analyzing the experimental results and considering the best of the cases, the SARIMA algorithm reduces the error to $7.8 \%$ when the observation time increases $177.1 \%$ for the high occupancy channel. The GARCH algorithm wanes in error about $15.3 \%$, with an increase in the observation time of $128 \%$ for the high occupancy channel. In both the ARIMA and GARCH algorithms, a training day to achieve acceptable prediction errors in the three GSM channels is sufficient according to the experimental results.

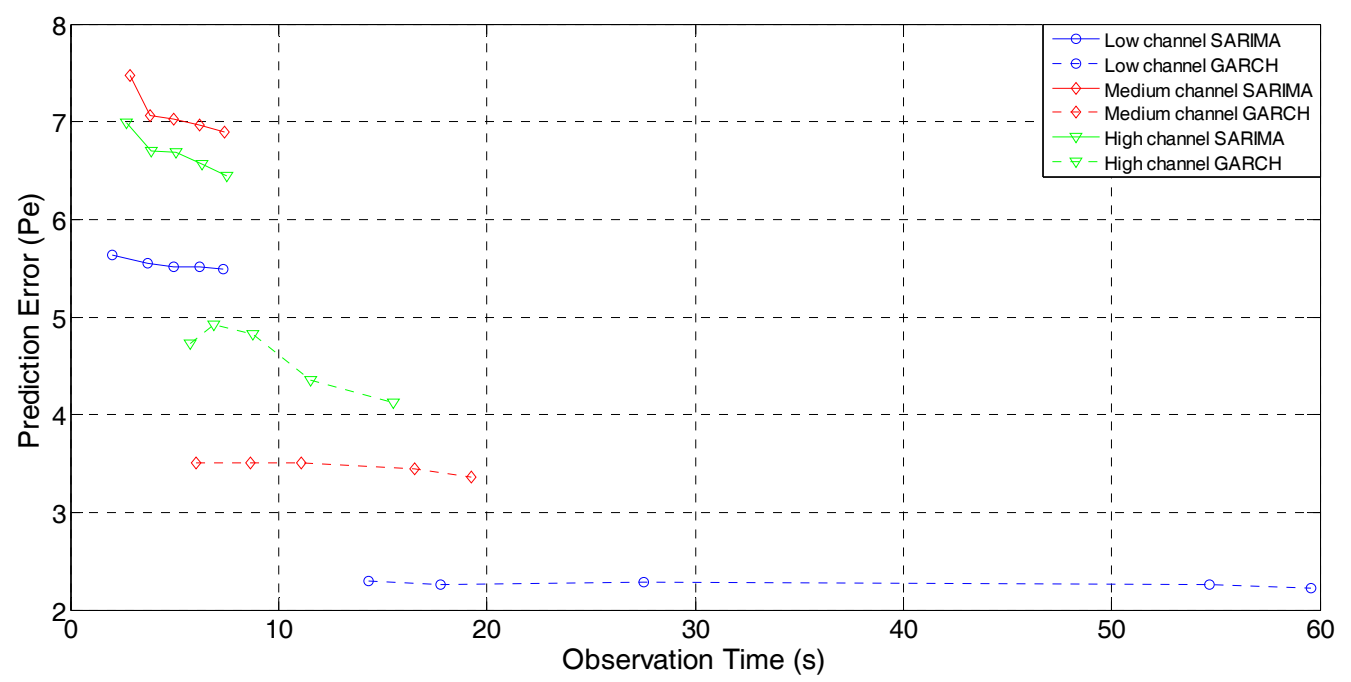

Figure 18. Prediction error vs. Observation time.

For the analysis of training, Tables 14-16 display the mean squared error for the forecast of reception power for the SARIMA and GARCH algorithms, with up to five days of training data. These results, and Figure 18, suggest that one training day in the SARIMA and GARCH algorithms is sufficient to obtain an acceptable error.

Table 14. Result of mean squared error for low channel with different number of training days.

\begin{tabular}{ccccc}
\hline $\begin{array}{c}\text { Number of } \\
\text { Training Days }\end{array}$ & $\begin{array}{c}\text { SARIMA Mean } \\
\text { Squared Error }\end{array}$ & $\begin{array}{c}\text { GARCH Mean } \\
\text { Squared Error }\end{array}$ & $\begin{array}{c}\text { SARIMA } \\
\text { Processing Time }\end{array}$ & $\begin{array}{c}\text { GARCH } \\
\text { Processing Time }\end{array}$ \\
\hline 1 & 18.64 & 25.48 & $3.88 \mathrm{~s}$ & $12.16 \mathrm{~s}$ \\
2 & 17.9 & 25.34 & $3.52 \mathrm{~s}$ & $18.57 \mathrm{~s}$ \\
3 & 18.29 & 24.51 & $4.25 \mathrm{~s}$ & $34.38 \mathrm{~s}$ \\
4 & 17.53 & 24.82 & $5.92 \mathrm{~s}$ & $41.42 \mathrm{~s}$ \\
5 & 17.17 & 23.76 & $7.65 \mathrm{~s}$ & $55.59 \mathrm{~s}$ \\
\hline
\end{tabular}

Table 15. Result of mean squared error for medium channel with different number of training days.

\begin{tabular}{ccccc}
\hline $\begin{array}{c}\text { Number of } \\
\text { Training Days }\end{array}$ & $\begin{array}{c}\text { SARIMA Mean } \\
\text { Squared Error }\end{array}$ & $\begin{array}{c}\text { GARCH Mean } \\
\text { Squared Error }\end{array}$ & $\begin{array}{c}\text { SARIMA } \\
\text { Processing Time }\end{array}$ & $\begin{array}{c}\text { GARCH } \\
\text { Processing Time }\end{array}$ \\
\hline 1 & 47.52 & 38.01 & $4.06 \mathrm{~s}$ & $8.61 \mathrm{~s}$ \\
2 & 45.19 & 38.37 & $4.28 \mathrm{~s}$ & $11.15 \mathrm{~s}$ \\
3 & 44.94 & 37.15 & $5.36 \mathrm{~s}$ & $11.38 \mathrm{~s}$ \\
4 & 42.38 & 36.68 & $6.73 \mathrm{~s}$ & $14.47 \mathrm{~s}$ \\
5 & 41.42 & 36.85 & $8.29 \mathrm{~s}$ & $18.55 \mathrm{~s}$ \\
\hline
\end{tabular}


Table 16. Result of mean squared error for high channel with different number of training days.

\begin{tabular}{ccccc}
\hline $\begin{array}{c}\text { Number of } \\
\text { Training Days }\end{array}$ & $\begin{array}{c}\text { SARIMA Mean } \\
\text { Squared Error }\end{array}$ & $\begin{array}{c}\text { GARCH Mean } \\
\text { Squared Error }\end{array}$ & $\begin{array}{c}\text { SARIMA } \\
\text { Processing Time }\end{array}$ & $\begin{array}{c}\text { GARCH } \\
\text { Processing Time }\end{array}$ \\
\hline 1 & 50.69 & 56.77 & $3.79 \mathrm{~s}$ & $7.57 \mathrm{~s}$ \\
2 & 48.35 & 59.8 & $3.96 \mathrm{~s}$ & $9.08 \mathrm{~s}$ \\
3 & 49.04 & 53.95 & $4.18 \mathrm{~s}$ & $10.61 \mathrm{~s}$ \\
4 & 46.51 & 51.56 & $4.82 \mathrm{~s}$ & $14.33 \mathrm{~s}$ \\
5 & 44.96 & 48.28 & $6.47 \mathrm{~s}$ & $15.83 \mathrm{~s}$ \\
\hline
\end{tabular}

\section{Conclusions}

The SARIMA and GARCH algorithms have been evaluated in this paper in order to forecast the reception power in channels of a GSM band. Even though GARCH algorithm presented lower prediction errors than SARIMA algorithm, the use of a SARIMA algorithm is more convenient for a CR system, because it has higher precisions with respect to availability and occupancy times, with which the use of spectrum efficiency is improved and the interference level and collisions between PUs and SUs will be reduced. Additionally, the SARIMA algorithm employs lower observation times than the GARCH algorithm. As noted, for the best of cases, observation times lower than four seconds could be obtained for the three GSM channels, which is an advantage in practical CR systems.

For a CR system, the forecast developed in the GSM band could help to improve the use of spectral efficiency, since it would allow CR users to share channels and avoid collisions with PUs in the found opportunities.

The SARIMA and GARCH algorithms forecast not only the reception power; but the occupation and availability times for GSM channels. It would also be feasible to use the training data from one day for the forecast of a CR user's received power from a primary BS.

The significance of the forecast of received power is that CR users can save energy in the process of detecting the spectrum and take advantage of spectral opportunities, thereby increasing the rate of successful transmission and transmission opportunities, reducing the time to find an available channel, and adjusting transmission power levels to protect against collisions and interference with the PUs.

Acknowledgments: This study was supported by Colciencias (Administrative Department of Science, Technology and Innovation of Colombia), Universidad Distrital Francisco José de Caldas and Universidad Nacional de Colombia.

Author Contributions: The SARIMA algorithm was developed by Luis Pedraza, GARCH algorithm was implemented by Cesar Hernandez, Ingrid Paez designed the presentation of results, Jorge Ortiz developed the results and Rodriguez-Colina analyzed the results. All authors read and approved the final manuscript.

Conflicts of Interest: The authors declare no conflict of interest.

\section{References}

1. Pedraza, L.; Forero, F.; Paez, I. Evaluación de ocupación del espectro radioeléctrico en Bogotá-Colombia. Ingeniería Ciencia 2014, 10, 127-143. (In Spanish) [CrossRef]

2. Pedraza, L.; Hernandez, C.; Paez, I. Evaluation of nonlinear forecasts for radioelectric spectrum. Int. J. Eng. Technol. 2016, 8, 1611-1626.

3. Gorcin, A.; Celebi, H.; Qaraqe, K.A.; Arslan, H. An autoregressive approach for spectrum occupancy modeling and prediction based on synchronous measurements. In Proceedings of the International Symposium on Personal Indoor and Mobile Radio Communications, Toronto, ON, Canada, 11-14 September 2011; pp. 705-709.

4. Pedraza, L. Redes Inalámbricas Mesh "Caso de Estudio: Ciudad Bolívar", 1st ed.; Universidad Distrital Francisco José de Caldas: Bogota, Colombia, 2012.

5. Pedraza, L.; Hernandez, C.; Galeano, K.; Rodriguez, E.; Paez, I. Ocupación Espectral y Modelo de Radio Cognitiva Para Bogotá, 1st ed.; Universidad Distrital Francisco José de Caldas: Bogota, Colombia, 2016. 
6. Wellens, M.; Riihijarvi, J.; Mahonen, P. Empirical time and frequency domain models of spectrum use. Phys. Commun. 2009, 2, 10-32. [CrossRef]

7. Song, C.; Chen, D.; Zhang, Q. Understand the predictability of wireless spectrum: A large-scale empirical study. In Proceedings of the International Conference on Communications, Cape Town, South Africa, 23-27 May 2010; pp. 1-5.

8. Sun, Z.; Laneman, J.N.; Bradford, G.J. Sequence detection algorithms for dynamic spectrum access networks. In Proceedings of the IEEE International Symposium on New Frontiers in Dynamic Spectrum, Singapore, 6-9 April 2010; pp. 1-9.

9. Fadda, M.; Popescu, V.; Murroni, M.; Angueira, P.; Morgade, J. On the feasibility of unlicensed communications in the TV white space: Field measurements in the UHF band. Int. J. Digit. Multimed. Broadcast. 2015, 2015, 1-8. [CrossRef]

10. Lopez, M.; Casadevall, F. Time-dimension models of spectrum usage for the analysis, design, and simulation of cognitive radio networks. IEEE Trans. Veh. Technol. 2013, 62, 2091-2104. [CrossRef]

11. Yarkan, S.; Arslan, H. Binary time series approach to spectrum prediction for cognitive radio. In Proceedings of the Vehicular Technology Conference, Baltimore, MD, USA, 30 September-3 October 2007; pp. 1563-1567.

12. Black, T.; Kerans, B.; Kerans, A. Implementation of hidden markov model spectrum prediction algorithm. In Proceedings of the International Symposium on Communications and Information Technologies, Gold Coast, Australia, 2-5 October 2012; pp. 280-283.

13. Li, Y.; Dong, Y.; Zhang, H.; Zhao, H.; Shi, H.; Zhao, X. Spectrum usage prediction based on high-order markov model for cognitive radio networks. In Proceedings of the International Conference on Computer and Information Technology, Bradford, UK, 29 June-1 July 2010; pp. 2784-2788.

14. Yu, C.; He, Y.; Quan, T. Frequency Spectrum Prediction Method Based on EMD and SVR. In Proceedings of the Intelligent Systems Design and Applications, Kaohsiung, Taiwan, 26-28 November 2008; pp. 39-44.

15. Wang, Z.; Salous, S. Time series arima model of spectrum occupancy for cognitive radio. In Proceedings of the Seminar on Cognitive Radio and Software Defined Radios: Technologies and Techniques, London, UK, 18 September 2008; pp. 1-4.

16. Chen, Y.; Oh, H.S. A Survey of Measurement-based Spectrum Occupancy Modeling for Cognitive Radios. IEEE Commun. Surv. Tutor. 2014, 1-36. [CrossRef]

17. Anand, N.C.; Scoglio, C.; Natarajan, B. GARCH-Non-linear time series model for traffic modeling and prediction. In Proceedings of the IEEE Network Operations and Management Symposium, Salvador, Brazil, 7-11 April 2008; pp. 694-697.

18. Tran, Q.; Ma, Z.; Li, H.; Trinh, Q. A Multiplicative Seasonal ARIMA/GARCH Model in EVN Traffic Prediction. Int. J. Commun. Netw. Syst. Sci. 2015, 8, 43-49. [CrossRef]

19. Zhanga, Y.; Fay, D.; Kilmartin, L.; Moore, A. A Garch-based adaptive playout delay algorithm for VoIP. Comput. Netw. 2010, 54, 3108-3122. [CrossRef]

20. Hyndman, R.; Koehler, A.B.; Ord, J.K.; Snyder, R.D. Forecasting with Exponential Smoothing: The State Space Approach, 1st ed.; Springer: Berlin/Heidelberg, Germany, 2008.

21. Stolojescu-Crisan, C. Data mining based wireless network traffic forecasting. In Proceedings of the International Symposium on Electronics and Telecommunications, Timisoara, Romania, 15-16 November 2012; pp. 115-118.

22. Hyndman, R.J. Another look at forecast-accuracy metrics for intermittent demand. Foresight Int. J. Appl. Forecast. 2006, 43-46.

23. Box, G.; Jenkins, G.; Reinsel, C. Time Series Analysis: Forecasting and Control, 4th ed.; Wiley: Hoboken, NJ, USA, 2008.

24. Tran, V.G.; Debusschere, V.; Bacha, S. Hourly server workload forecasting up to 168 hours ahead using Seasonal ARIMA model. In Proceedings of the IEEE International Conference on Industrial Technology, Athens, Greece, 19-21 March 2012; pp. 1127-1131.

25. Permanasari, A.E.; Hidayah, I.; Bustoni, I. SARIMA (Seasonal ARIMA) implementation on time series to forecast the number of Malaria incidence. In Proceedings of the International Conference on Information Technology and Electrical Engineering, Yogyakarta, Indonesia, 7-8 October 2013; pp. 203-207.

26. Pedraza, L.; Hernandez, C.; Rodriguez, E. Modeling of GSM Spectrum Based on Seasonal ARIMA model. In Proceedings of the 6th IEEE Latin-American Conference on Communications, Cartagena, Colombia, 5-7 November 2014; pp. 1-4. 
27. Engle, R. The Use of ARCH/GARCH Models in Applied Econometrics. J. Econ. Perspect. 1982, 15, 157-168. [CrossRef]

28. Bollersleva, T. Generalized autoregressive conditional heteroskedasticity. J. Econom. 1986, 31, 307-327. [CrossRef]

29. Chinomona, A. Time Series Modelling with Application to South African Inflation Data. Master's Thesis, University of KwaZulu-Natal, Pietermaritzburg, South Africa, 2009.

30. Edward, N. Modelling and Forecasting Using Time Series Garch Models: An Application of Tanzania Inflation Rate Data. Master's Thesis, University of Dar es-Salaam, Dar es Salaam, Tanzania, 2011.

31. Talke, I. Modelling Volatility in Time Series Data. Master's Thesis, University of KwaZulu-Natal, Pietermaritzburg, South Africa, 2003.

32. Pedraza, L.; Forero, F.; Paez, I. Metropolitan Spectrum Survey in Bogota Colombia. In Proceedings of the IEEE International Conference on Advanced Information Networking and Applications Workshops, Barcelona, Spain, 25-28 March 2013; pp. 548-553.

33. Lopez, M.; Casadevall, F. Methodological aspects of spectrum occupancy evaluation in the context of cognitive radio. Eur. Trans. Telecommun. 2010, 21, 680-693. [CrossRef]

34. International Telecommunication Union. Report ITU-R SM.2256, Spectrum Occupancy Measurements and Evaluation; ITU: Geneva, Switzerland, 2012.

35. Digham, F.; Alouini, M.S.; Simon, M. On the Energy Detection of Unknown Signals Over Fading Channels. IEEE Trans. Commun. 2007, 55, 21-24. [CrossRef]

36. Dickey, D.A.; Fuller, W.A. Distribution of estimators for autoregressive time series with a unit root. J. Am. Stat. Assoc. 1979, 74, 427-431.

37. Bozdogan, H. Model selection and Akaike's Information Criterion (AIC): The general theory and its analytical extensions. Psychometrika 1987, 52, 345-370. [CrossRef]

(C) 2016 by the authors; licensee MDPI, Basel, Switzerland. This article is an open access article distributed under the terms and conditions of the Creative Commons Attribution (CC-BY) license (http://creativecommons.org/licenses/by/4.0/). 